\title{
Efficacy and Safety of Treatment with Quadruple Oral Hypoglycemic Agents in Uncontrolled Type 2 Diabetes Mellitus: A Multi-Center, Retrospective, Observational Study (Diabetes Metab J 2021;45:675-83)
}

Tae Jung Oh

Department of Internal Medicine, Seoul National University Bundang Hospital, Seoul National University College of Medicine, Seongnam, Korea

There are numerous reasons for clinical inertia to insulin, such as fear of injection, negative appraisals of insulin, and side effects [1]. From the data of Diabetes Fact Sheets in Korea 2020 [2], the percentage of patients with type 2 diabetes mellitus using insulin is only $4.1 \%$, much lower than the $15 \%$ estimated in UK data in 2010 [3]. Instead, many physicians prescribe quadruple oral hypoglycemic agents, which are not approved by the Korea Food and Drug Administration. Therefore, the efficacy and safety of this regimen must be analyzed.

In this article entitled, "Efficacy and safety of treatment with quadruple oral hypoglycemic agents in uncontrolled type 2 diabetes mellitus: a multi-center, retrospective, observational study," Moon et al. [4] reported clinical data of efficacy and safety of quadruple oral hypoglycemic agents in the Yeongnam and Honam regions of Korea. This multicenter study showed a $75.6 \%$ adherence rate to quadruple oral hypoglycemic agents for 12 months, producing a $-1.1 \% \pm 1.2 \%$ reduction of glycosylated hemoglobin (HbAlc) without any significant safety issue. The HbAlc-lowering efficacy was similar when including patients who did not continue the quadruple regimen despite no reported reason for discontinuation. As a higher baseline HbAlc $(\geq 9.0 \%)$ represents better glycemic lowering effect after quadruple therapy [5], a good response is expected in the current study because the baseline HbAlc was $9.0 \% \pm 1.3 \%$. Even though observational studies $[4,5]$ have shown the effec- tiveness of the quadruple regimen, further randomized clinical trials are needed to compare this regimen with placebo or injection therapy.

There is doubt about the short- and long-term safety of the quadruple regimen. The hypoglycemia in this study was captured only by electronic records from an emergency room visit. However, most hypoglycemic events occur outside of the healthcare system [6]. The present study did not report any hypoglycemic events, even though $94.4 \%$ of patients were treated with sulfonylurea. Therefore, underestimation of hypoglycemia is suspected, and the safety of quadruple oral hypoglycemic agents regarding hypoglycemia remains unknown. Furthermore, $74.8 \%$ of patients had not achieved the treatment target (HbAlc $<7.0 \%$ ). Therefore, diabetic vascular complications might not be effectively prevented in the majority of patients under quadruple regimen. In addition, baseline renal function was relatively well-preserved, with mean estimated glomerular filtration rate of $90.0 \mathrm{~mL} / \mathrm{min} / 1.73 \mathrm{~m}^{2}$. The indications of metformin and sodium-glucose co-transporter 2 inhibitor for renal impairment are broadening, and the quadruple regimen must be studied in patients with renal impairment. The current study did not involve clinical data of quadruple regimen use in patients with renal impairment.

In my opinion, the authors have reported valuable results about real clinical data of quadruple regimens. However, as the
Corresponding author: Tae Jung Oh (iD https://orcid.org/0000-0002-5078-6123 Department of Internal Medicine, Seoul National University Bundang Hospital, Seoul National University College of Medicine, 82 Gumi-ro 173beon-gil, Bundang-gu, Seongnam 13620, Korea

E-mail: ohtjmd@gmail.com
This is an Open Access article distributed under the terms of the Creative Commons Attribution Non-Commercial License (https://creativecommons.org/licenses/by-nc/4.0/) which permits unrestricted non-commercial use, distribution, and reproduction in any medium, provided the original work is properly cited. 
authors mentioned in the last sentence in their article, these data do not rule out the need for insulin therapy.

\section{CONFLICTS OF INTEREST}

No potential conflict of interest relevant to this article was reported.

\section{REFERENCES}

1. Russell-Jones D, Pouwer F, Khunti K. Identification of barriers to insulin therapy and approaches to overcoming them. Diabetes Obes Metab 2018;20:488-96.

2. Jung CH, Son JW, Kang S, Kim WJ, Kim HS, Kim HS, et al. Diabetes fact sheets in Korea, 2020: an appraisal of current status. Diabetes Metab J 2021;45:1-10.
3. Holden SE, Gale EA, Jenkins-Jones S, Currie CJ. How many people inject insulin?: UK estimates from 1991 to 2010. Diabetes Obes Metab 2014;16:553-9.

4. Moon JS, Suh S, Kim SS, Jin HY, Kim JM, Jang MH, et al. Efficacy and safety of treatment with quadruple oral hypoglycemic agents in uncontrolled type 2 diabetes mellitus: a multi-center, retrospective, observational study. Diabetes Metab J 2021;45: 675-83.

5. Cho YK, Lee J, Kim HS, Park JY, Jung CH, Lee WJ. Clinical efficacy of quadruple oral therapy for type 2 diabetes in realworld practice: a retrospective observational study. Diabetes Ther 2020;11:2029-39.

6. Karter AJ, Moffet HH, Liu JY, Lipska KJ. Surveillance of hypoglycemia: limitations of emergency department and hospital utilization data. JAMA Intern Med 2018;178:987-8. 